\title{
Capric Acid
}

National Cancer Institute

\section{Source}

National Cancer Institute. Capric Acid. NCI Thesaurus. Code C68329.

A saturated medium-chain fatty acid with a 10-carbon backbone. Capric acid is found naturally in the coconut and palm kernel oils as well as the milk of various mammals. 